\title{
LCA Research at the Swiss Federal Institute of Technology (EPFL and ETHZ)
}

\author{
Gerald Rebitzer ${ }^{1 *}$, Andrea Weber Marin ${ }^{2}$ and Olivier Jolliet ${ }^{1}$ \\ ${ }^{1}$ Swiss Federal Institute of Technology, Group for Life Cycle Systems, EPFL, CH-1015 Lausanne, Switzerland \\ ${ }^{2}$ Swiss Federal Institute of Technology, Institute for Manufacturing Automation, CH-8092 Zürich, Switzerland \\ * Corresponding author (Gerald.Rebitzer@epfl.ch)
}

The Swiss Federal Institute of Technology has been in the forefront of LCA research since the beginning of this discipline. Today, two of the pillars of this national research and educational organization, located in Zürich (Eidgenössische Technische Hochschule Zürich - ETHZ) and Lausanne (École Polytechnique Fédérale de Lausanne-EPFL), continue this tradition and are prominent centres of LCA activities in Europe.

In Lausanne, there have been two groups focusing on LCA and life cycle management (LCM) research, as well as several other groups who have applied LCA methodologies (e.g. in the development of energy systems). Beginning in March 2002, the main LCA and LCM-related research at the EPFL will be conducted under the single umbrella of the Group for Life Cycle Systems (http://gecos.epfl.ch/lcsystems), headed by Olivier Jolliet (Olivier.Jolliet@epfl.ch)'

LCA research activities at the ETHZ are distributed over different institutes, which are connected to the department of Environmental Sciences or to engineering departments. Mainly small groups or single researchers within these institutes focus on LCA development or application. For information exchange and discussion on LCA-topics or single projects, those researchers meet in the 'LCA-Werkstätte' which is organized periodically (information at Werkstatt@tech.chem.ethz.ch).

The following short synopses provide insights into some of the recent and ongoing research activities at ETHZ and EPFL. These activities cover a wide spectrum of research related to system modelling and life cycle inventory issues, life cycle impact assessment methodology, as well as application of LCA and life cycle management. In the following, an excerpt is presented which is structured by the themes mentioned above.

A Structured Model for Quantitative Analysis of Production Systems (Andrea Weber Marin, ETHZ, Weber@produktion.bepr. ethz.ch): Complex industrial production systems require life cycle tools that are able to handle a large number of independent process units. Structured Analysis is a graphical method based on three static and dynamic diagram types. It was designed for software development, but is now adapted for the handling of resources like energy, human workforce, and costs. Structured Analysis helps to visualize the system on several hierarchical levels, while facilitating a bottom-up or a top-down approach. By applying this methodology, enriched by an exergy/ energy balance, a human workforce analysis, and a cost calculation, new insights can be gained concerning technical solu-

' The former group of David Hunkeler will be incorporated into this life cycle group, while David Hunkeler himself will leave the EPFL to expand his business ventures in wastewater treatment and life cycle management. tions for energy reuse and alterations in work priority and costing. The dynamic diagrams enable the step to discrete simulation for alternative production scenarios.

Scenario Construction for Strategic LCA (Michael Spielmann, ETHZ, Spielman@uns.umnw.ethz.ch): The environmental assessment of a new high-speed transport technology (Swissmetro) compared to alternative means of transport serves as an example for a multi-level scenario analysis that distinguishes Service Scenarios-addressing options and uncertainties in the use phase - and Life Cycle Scenarios - addressing alternatives and uncertainties in the upstream and downstream processes. Service Scenarios and Life Cycle Scenarios are jointly evaluated using a formative scenario analysis, resulting in a set of impact factors with different combinations of levels. Each combination represents a scenario of a possible future product system. All combinations are checked for consistency and a representative set of a few consistent product system scenarios is selected. The developed scenario procedure emerges as a promising method for strategic LCA studies.

Modelling System Boundaries in Simplified LCAs (Gerald Rebitzer, EPFL, Gerald.Rebitzer@epfl.ch): For many applications, the time and cost requirements of an LCA are not considered to correspond to the possible benefits of the results by many, if not most, decision makers. These limitations are particularly acute within contexts where a rapid decision for a new product or technology is required, such as during a design for environment (DfE) process. In this context, the system boundaries of an LCA play a crucial role, since they determine the size and complexity of the product system to be assessed. This research therefore focuses on methods for simplifying LCA by restricting the system boundaries. Among other approaches, different uses of mass and energy cut-off criteria are explored. Preliminary research from an automotive application suggests that process-specific mass cut-offs in the range of 6-8\% might be suitable for many DfE applications. In addition to cases from the automotive sector, an LCA for wastewater treatment options is underway and also serves as a basis for simplification investigations.

Environmental Life Cycle Impacts of Internet - Combining Process LCA and Input-Output LCA (Yves Loerincik, EPFL, Yves. Loerincik@epfl.ch; Filippo Della Croce, EPFL, Filippo.Dellacroce@ epfl.ch): The objective of this study is to assess the environmental life cycle impacts of the internet infrastructure by looking at a local university network and the overall US infrastructure using two approaches to life cycle assessment: Process LCA and Input-Output LCA (IO-LCA). Both approaches show that the PCs (control units and screens) are dominating the system's energy use and $\mathrm{CO}_{2}$ emissions. One has to note that the em- 
bodied energy consumption for infrastructure production plays an increasingly important role with the use of notebooks or flat screens. The overall results (considered impacts) of the IO-LCA were a factor of 2 to 4.5 larger than the results of the Process LCA. The main reason for this difference is the larger number of inputs considered by an IO-LCA (mainly air transportation and small contributions such as business-related lodging, legal services, and banking), though on a less specific level. Therefore, a hybrid approach is being developed in order to take advantages of both approaches. The analysis further expands the boundaries, showing the significant environmental burdens of non-hardware related input requirements, such as software development, training, management, trouble-shooting. In addition, a preliminary assessment of the potential reduction in emissions induced by the introduction of e-commerce is provided (wholesale and retail are responsible for $14 \%$ of the total U.S. energy consumption).

Assessment of Toxicological Impacts (David Pennington, EPFL, David.Pennington@epfl.ch): This research focuses on the characterization of the time and space-integrated likelihood and potential consequences of toxicological impacts associated with low-level/disperse chemical emissions. Such indicators are beneficial in the comparison of chemicals and emissions in application domains such as chemical screening, product life cycle assessment (LCA), and in preferential purchasing programmes. Specific activities include the development of EPFL's tool 'Impact assessment of chemical toxicants' (IMPACT 2002, download at http://gecos.epfl.ch/lcsystems; includes Disability Adjusted Life Years (DALYs) for more than 900 carcinogens and non-carcinogens as based on $\mathrm{ED}_{10 \mathrm{~h}}$, the effect dose inducing a $10 \%$ added risk for humans), collaboration in the European Union's project 'Operation models and information tools for industrial applications of eco/toxicological impact assessments' (OMNIITOX), co-development of spatial multimedia chemical fate and human exposure tools for Europe, and contributions to Japan's National Life Cycle Assessment Initiative.

Spatial Fate and Exposure Modelling (Christoph Ammann, EPFL, David.Pennington@epfl.ch): Spatial variation is not taken into account in many characterization factors in LCIA. Building on a prototype model for Japan developed by David Pennington, this research focuses on the development of a spatial multimedia model to estimate the fate and human exposure of chemicals in Western Europe. The model takes into account watershed boundaries, advective airflow patterns, food production distribution, population density, and water sources. Preliminary results indicate that, especially for pollutants that predominantly partition into water, the influence of spatial variation can be very important - up to a factor of 4000 variation in human exposure has been noted for certain chemicals. Whether multimedia models without spatial resolution can provide reliable estimates of even mean characterization factors for toxicological impacts is questioned.

Fate \& Exposure Modelling in LCIA (Manuele Margni, EPFL, Manuele.Margni@epfl.ch): Margni developed a modular approach to calculate the multimedia fate and multi-pathway exposure of organic chemicals for use in life cycle and risk assessment. The model enables the estimation of median intake fractions (fraction of pollutant released into the environment that results in human exposure at a population level) through the different pathways, taking into account food production levels, water supply, and inhalation. Modularisation helps im- prove transparency in existing multimedia approaches, whilst opening the door to the combination of robust single-medium models. Prototypes have been developed for Europe and Japan. These prototypes help facilitate the calculation of site-dependent, as well as generic, toxicological characterization factors for humans and ecosystems in EPFL's LCIA tool 'IMPACT 2002' (Impact Assessment for Chemical Toxicants: A modular approach for the assessment of chemical fate, exposure, and toxicological impacts; see above).

Development of the AMI Method for the Assessment of the Median Impact of Chemicals on Ecosystems in LCIA (Jerome Payet, EPFL, Jerome.Payet@epfl.ch): The comparative assessment of the impacts of chemicals on ecosystems requires improvements in terms of several methodological aspects, like the selection of the data used for the assessment, and the robustness of the ecotoxicity indicators. At the same time, it is necessary to use a method that is as transparent as possible and which yields readily interpretable results. To reach these objectives, the AMI (Assessment of the Median Impact) method is based on three key principles. (1) Ecotoxicological endpoint: The method is based on single species laboratory test $\mathrm{EC}_{50}$ (Effect concentration for $50 \%$ of species) results - the endpoint with the lowest uncertainty and which enables validation of the models used in LCIA; (2) The median based estimator is more appropriate when a specific distribution cannot be assumed; (3) Calculation of the uncertainty of the ecotoxicity indicator is based on a (non-parametric) bootstrap method. The AMI method currently facilitates the calculation of characterization factors for about 500 chemicals, with associated parameter uncertainty measures.

LCA of Pesticides in Plant and Agriculture (Raphaël Charles, EPFL and Swiss Federal Research Station for Plant Production, Raphael.Charles@epfl.ch): This research aims to develop a methodology for environmental impact assessment of pesticides applied to arable crops. Improvements are expected in terms of better reliability and acceptance of the LCA methodology for chemicals used in agriculture applications. Going from detailed models, the functioning of the complex system phytosanitary measures - plant - environment is studied. A first model has been developed to provide insights into the efficiency of pesticide transfer from air, soil, and deposition to the leaves to the plant. Essential parameters are identified as Octanol-Water Partitioning Coefficient (Kow) and the half-life of the pesticides. Agronomic and practical characteristics are also integrated in the latest developments of the method.

LCA of Agricultural Chemicals (Georg Geisler, ETHZ, Geisler@ tech.chem.ethz.ch): Modern agrochemicals feature high specificity and low doses in use, but also complex molecular structures causing high production efforts. In an LCA of an older and a modern plant growth regulator, environmental effects from production and application are compared. The inventory of chemical production is modelled in best and worst-case scenarios, based on stoichiometric measures and generic utility demands. The low dose of the modern agrochemical compensates for the high environmental effects from its production. However, the old plant growth regulator shows overall trends towards less environmental impacts than the new one, but no significantly different results for the two substances are obtained to date. Thus, improvement options for the inventory model will be analysed. 
A Spatial Source/Sink Concept for the Assessment of Environmental Effects of Primary Production Systems on a Single Scale as a Basis for Decision-Making (Regina Brandenberger, ETHZ, Brandenberger@fowi.ethz.ch): Renewable primary products have a competitive disadvantage if land use is assessed only based on direct production area. Land use must therefore be balanced against other environmental impacts. This is achieved by extending the input-output inventory of a traditional LCA with a source/sink concept, where impacts are based on the source and sink capacity that is required to generate goods or offset associated emissions (similar to the Ecological footprint concept). The methodology uses space as a single, physically meaningful, measurement scale and can also be used as a basis for decision-making.

Life Cycle Assessment of Biofibres and of Polymers Produced by Plants (Mathieu Thérézien, EPFL, Mathieu.Therezien@epfl.ch): The objectives of this study are to evaluate whether the substitution of glass fibres by natural fibres or of conventional fossil oil-based plastics by biopolymers (PHBV) is interesting from an environmental perspective. In addition, the key parameters in the production of these novel materials are identified. An LCA has been performed on China reed fibre used as a substitute for glass fibre reinforcement in plastics for an application in transport pallets. Transport pallets reinforced with China reed fibre prove to be ecologically advantageous due to a) the substitution of glass fibre production by the natural fibre production, b) the indirect reduction in the use of polypropylene linked to the higher proportion of China reed fibre used, and c) the reduced pallet weight, which reduces fuel consumption during transport. An LCA of PHBV [poly(3 hydroxybutyrate co 3 hydroxyvalerate)] polymers used for plastic bottles shows that PHBV extracted from bacteria is likely to have higher impacts on the environment than its petrochemical counterparts due to glucose production from corn and heating requirements throughout the process. Extracting PHBV from colza promises a more environmentally friendly solution, provided that solvent losses during extraction processes are minimized.

LCA of Cotton Growing in the Texas High Plains (Simone Schärer, ETHZ, Schaerer@hotmail.com): Five cotton cultivation scenarios were selected to be investigated and compared by LCA, including organic, dry-land, conventional, and genetically modified cotton. The evaluation of the inventory data was performed with the LCA software tool SimaPro. Here, in addition to the critical surface time (CST) evaluation method used by Margni (EPFL, Group for Life Cycle Systems), the inventory was also assessed with the method of the Center voor Milieukunde (CML). All investigations show that the organic growing scenario has a negligible influence on the environment compared to the other growing scenarios. In the conventional growing scenarios, the toxicological effects of the active ingredients of the pesticides used lead to dominant environmental effects in the categories of 'terrestrial ecotoxicity' and 'aquatic ecotoxicity'.

Waste Management of Electronic Products in Switzerland (Daniel Oetiker, ETHZ; Marion Tobler, ETHZ, Tobler@produktion. bepr.ethz.ch): Forced by the pressure of legislation (e.g. the EU directive proposal 'Waste from Electrical and Electronic Equipment' - WEEE), the European electronics industry breaks new grounds for waste treatment of electronic equipment. This study investigates the environmental impacts caused by implementing recycling technology compared to incineration. Material flows of electronics waste, recycling process technology, and economics are analysed in a case study. Two different compositions of material were chosen: general electronics waste and scrapped copy machines. The impacts were calculated by means of different LCA methods, EI99, EI95, EP and CML, with the LCA software SimaPro. The main finding was that recycling is the superior solution, confirmed by all methods, with different values of impact ratio. Treatment cost for electronics waste in incineration, however, are more than $30 \%$ lower.

Waste Solvent Management in Swiss Chemical Industry (Christina Seyler-Jahn, ETHZ, Jahn@tech.chem.ethz.ch): Large amounts of spent solvent arise in the chemical industry every day. The solvent-to-product ratio can be as high as 100 for pharmaceutical products. As pharmaceuticals are produced batch-wise in multipurpose plants, the waste solvent management cannot be optimised in the same way as for continuous production processes in single-purpose plants. Therefore, decisions on what to do with the waste solvent have to be made on a weekly basis. There are two treatment options common in the chemical industry: distillation and thermal treatment. In distillation, the solvent is recovered but energy is used for the process. In thermal treatment, the energy content of the solvents is recovered but new solvent has to be produced. This thesis compares these two treatment options by using LCA methodology.

Development of an Environmental and Socio-Economic Assessment Method for Local Authorities (Tourane Corbière-Nicollier, EPFL, Tourane.Corbiere@epfl.ch): This study aims at developing a straightforward tool, usable at the commune level, to determine the socio-economic and environmental impacts of various alternatives in order to establish sustainable development priorities. The research relies on a life-cycle thinking approach, applying both Process and Input-Output LCA and differentiating onsite (local) from life cycle impacts. One of the initial key performance indicators for a first screening is the primary nonrenewable energy used, provocatively expressed in 'energy slaves' (3150 MJ/year = 1 energy slave, which relates to constantly producing 200 Watts for 12 hours a day - an image of a human energy producer, e.g. on a bicycle). An average Swiss citizen needs 20 slaves for his private consumption of goods and services, 19 for housing, 11 for transport, 11 for food and 8 are for his or her consumption of public goods. A comparable approach is being developed to determine the environmental life cycle and economic performances of a company as a whole.

The Influence of Structural Changes in the Economy on the Consumption of Resources (Christine Najar, ETHZ, Najar@se. baug.ethz.ch): In order to be able to answer questions concerning the effects of resource taxes and other political instruments, as well as political developments and new technologies, it is necessary to analyse the combination of economic and ecological methods. Naming just a few, there are Input-Output Analysis, LCA and Material Flux Analysis (MFA). This thesis scrutinizes the construction industry in Switzerland. How were the uses of resources such as wood, lime, and marl influenced by developments in the past and how will they be used in the future? Another point of interest is the impact of political instruments or technology on the construction industry itself. This, for instance, is concerned with industry's typical features in Switzerland compared to other countries like the Netherlands, Germany, or the USA. 